\title{
Two novel, severe asthma phenotypes identified during childhood using a clustering approach
}

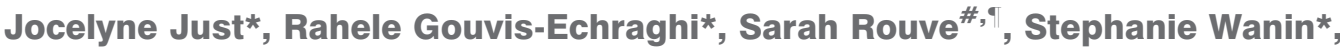 \\ David Moreau" ${ }^{\#}$ and Isabella Annesi-Maesano ${ }^{\#, 9}$
}

ABSTRACT: Unsupervised cluster analysis has already been used to identify severe phenotypes of childhood asthma, but without taking into account inflammatory markers. The aim of this study was to define independent homogeneous phenotypic clusters of severe asthma in a cohort of asthmatic children.

Cluster analysis was applied to 19 variables from 315 children enrolled in the Trousseau Asthma Program in Paris, France.

Three independent clusters of asthma were identified. Cluster 1, asthma with severe exacerbations and multiple allergies: 103 children had more sensitisations to inhaled allergens and food allergens, more blood eosinophils and basophils, more uncontrolled asthma despite high doses of inhaled corticosteroid and more hospitalisations for exacerbation. Cluster 2, severe asthma with bronchial obstruction: 72 children were significantly older, had the highest body mass index, a lower forced expiratory volume in $1 \mathrm{~s}$, more pronounced blood neutrophils and significantly higher levels of all classes of immunoglobulin (Ig), except IgE. Cluster 3, mild asthma: 140 children did not show statistically significant features.

These results could lead to improved management of severe asthma in children by optimising treatment strategies, i.e. anti-allergic drugs, such as anti-IgE for children with the allergic phenotype, and anti-neutrophil drugs, such as macrolides for those with the obstructive phenotype.

KEYWORDS: Allergic inflammation and asthma, childhood asthma, cohort study, immunoglobulin



sthma constitutes a growing public health problem due to the increasing prevalence over the past decades, particularly among young children and in emerging countries [1]. In addition, severe asthma is the leading cause of hospitalisation among preschool children and this explains, in part, that the cost associated with this disease is largely related to the most severe forms by the indirect costs it generates [2].

Asthma is no longer considered as just one disease but rather a complex of multiple and variously associated syndromes expressing different phenotypes [3]. In children, asthma is characterised by a different prognosis according to the age of onset [4]. The Tucson birth cohort [5] identified a phenotype of early-onset asthma which persists in childhood. Children presenting with this phenotype have impaired lung function at school age whereas their lung function in early childhood was comparable to that of children who never wheeze. This phenotype has a strong familial component, is predominantly allergic [6] and is often associated with more severe disease [7]. For preschool children, other severe asthma phenotypes have been described [8], such as "intermittent severe wheezing", often associated with allergic manifestations, and "multiple trigger wheezing", with a poorer long-term prognosis than other types of wheeze. The clustering approach has been used in cases of severe asthma to identify novel phenotypes. In this respect, most studies to date have been conducted on adults, although a recent study identified four phenotypes of asthma in school-age children through cluster analysis according to the severity of obstructive airway disease [9].

In conclusion, phenotyping of childhood asthma has rarely been based on cluster analysis and features other than atopic status and lung function level, in spite of the clinical characteristics of the asthmatic condition in children. However, asthma is also characterised by inflammation of
AFFILIATIONS

${ }^{*}$ Centre de l'Asthme et des Allergies, Hôpital d'Enfants Armand-Trousseau, \#INSERM, U 707 EPAR, and "UPMC Univ Paris 06, Medical School Saint-Antoine, UMR S 707 EPAR, Paris, France.

CORRESPONDENCE

J. Just

Centre de l'Asthme et des Allergies Groupe Hospitalier Trousseau - La Roche Guyon

26 avenue du Docteur Arnold Netter 75012 Paris

France

E-mail: jocelyne.just@trs.aphp.fr

Received:

July 192011

Accepted after revision:

Oct 262011

First published online:

Jan 202012 
the air passages resulting in the temporary narrowing of the airways. The purpose of our study was to apply an unsupervised analytical approach to distinguish complex asthma phenotypes without $a$ priori definitions of the condition on the hypothesis that in school age children, asthma is divided into phenotypes depending on severity and related not only to atopic status and airways obstruction but also to the underlying inflammatory mechanisms.

\section{METHODS}

Patients were part of the 9-yr (1998-2009) Trousseau Asthma Program of the "Centre de l'Asthme" at the Hôpital Trousseau in Paris, France, which included children aged 6-12 yrs suffering from severe asthma. Two-thirds of the children were outpatients from Paris and the surrounding area and the rest of them were from regions throughout France and were referred to the centre by a primary care physician due to persistent asthma. The children's data were collected in a computerised database using questionnaires and clinical examinations following a standardised protocol for all children.

The population analysed in the present study consists of all children meeting the following inclusion criteria: 1) aged 612 yrs at the time of exploration; 2 ) a history of $a \geqslant 12 \%$ change in forced expiratory volume in $1 \mathrm{~s}$ (FEV1) after bronchodilator administration; 3) meeting the criteria for persistent asthma [10]; 4) any chronic obstructive pulmonary disease (congenital or acquired origin) other than asthma had been ruled out; and 5) undergoing clinical examinations outside of episodes of exacerbation or acute respiratory illness.

\section{Cluster analysis parameters}

The parameters included in the cluster analysis were as follows. 1) Age and body mass index (BMI) measured during the medical visit. 2) Asthma duration in two classes: $<5$ or $>5$ yrs of age. 3) Maternal or paternal asthma. 4) Allergic sensitisation to aeroallergens and trophoallergens defined by a positive skin-prick test (SPT; wheal allergen $\geqslant 3 \mathrm{~mm}$ in the absence of a positive reaction to the negative control), and confirmed by positive specific immunoglobulin (Ig)E $\left(\geqslant 0.35 \mathrm{kU} \cdot \mathrm{L}^{-1}\right)$ (ImmunoCAP ${ }_{\circledR} ;$ Phadia, Uppsala, Sweden). The following standardised inhaled allergens and food allergens were administered: house dust mites, cat and dog dander, grass and birch pollen, Alternaria, cow's milk, egg, peanut, wheat and fish. 5) Total IgE level $\left(\mathrm{kU} \cdot \mathrm{L}^{-1}\right)$. 6) Inflammatory markers measured in peripheral blood through lymphocyte, neutrophil, eosinophil, basophil, and monocyte counts expressed in absolute rate (cell counting by automated Sysmex ; Roche Diagnostics, Roissy, France) and serum IgG, IgA and IgM levels $\left(\mathrm{g} \cdot \mathrm{L}^{-1}\right)$ using the immunoturbidimetry technique (PLC Modular ${ }^{\circledR}$; Roche Diagnostics, Meylan, France). 7) Lung function measured by spirometry (SpiroDyn'R $R_{\mathbb{R}}$; Dyn'R, Muret, France) according to the American Thoracic Society/European Respiratory Society recommendations [11]. Baseline FEV1 was expressed as a percentage of the predicted value with respect to sex, size and weight. 8) Severity (mild persistent, moderate persistent and severe persistent) and control (controlled or partially and uncontrolled) were assessed according to the Global Initiative for Asthma (GINA) guidelines [12]. All enrolled children were on a stable dose of inhaled corticosteroids (ICS) for $\geqslant 6$ months and were compliant with their prescribed asthma treatment.
Thresholds for high-dose ICS were defined as $\geqslant 500 \mu \mathrm{g}$ fluticasone (or equivalent) per day [10]. Asthma control was assessed in four classes: controlled with or without high-dose ICS, or uncontrolled (including partially controlled) with or without high-dose of ICS. Finally, severe exacerbation, defined as at least one hospitalisation for asthma exacerbations, was also recorded.

\section{Variable reduction and data transformation}

The initial dataset provided almost 40 variables selected from a large spectrum of routine assessments of asthmatic children. The following a priori strategies were used to reduce the number of variables before performing the cluster analysis. Missing variables were excluded. The number of variables that were clinically redundant was reduced by selecting variables reflecting major physiological parameters, e.g. pre- and postbronchodilator FEV1, basal FEV1 was only included in cluster analysis. Data from the questionnaires were excluded if the data were presented in the text (such as the name of the ICS), or if the information would have been irrelevant for the current analysis (such as the type of dwelling or parental race). A spectrum of response variables were transformed into "composite variables", e.g. positive allergen SPTs were transformed into number of sensitisations to food allergens or inhaled allergens. In addition, variables that correlated with each other were mutually excluded from the model to avoid bias, e.g. FEV1 and forced expiratory flow at $25-75 \%$ of forced vital capacity.

The principal component analysis was then used to select the final variables to be included in the model according to statistical significance; resulting in 19 variables. Subjects were required to have all of the 19 variables. Demographic data (age and $\mathrm{BMI}$ ), additional variables previously reported to have an effect on disease severity (asthma duration), elements of current classification schemes (medication use) or risk (hospitalisation for asthma exacerbation), and parameters with important physiological measurements (lung function and atopy) were included in the cluster analysis.

Despite the fact that our cluster analysis was unsupervised, the stability of the clusters was tested using data resampling techniques, by randomly drawing the variables from the model, and by altering the sample or subsetting [13].

\section{Statistical analysis}

All available variables were included in the statistical analysis. Data standardisation was performed before beginning analyses. The k-means method (the FASTCLUS procedure in SAS version 9.2; SAS Institute Inc., Cary, NC, USA) was used as preliminary analysis to produce a large number of disjoint clusters of observations. Hierarchical cluster analysis was then performed to hierarchically cluster these preliminary clusters using Ward's minimum variance method (the CLUSTER procedure, SAS 9.2). Categorical variables between the different groups of asthma severity and clusters were compared with the Chi-squared test or Fisher's exact test, respectively, when the required conditions were not respected. In the case of continuous variables, groups were compared with the t-test and one-way ANOVA when the hypotheses of B normality and variance equality were confirmed and otherwise with the Wilcoxon and Kruskal-Wallis test. The Tukey and Dunn tests 
were used for post hoc multiple comparisons [14]. The 95\% confidence interval of the mean rather than the standard deviation was introduced to better investigate whether there was an overlap of the variable values among the clusters. All analyses were performed with SAS version 9.2. For each bilateral test, type I error was taken to be $5 \%$.

\section{Ethics}

The parents of each child provided written informed consent before the questionnaire was distributed. As all procedures reflect common patient care at the study centre, the protocol was endorsed by the Institutional Review Board of the Medical Ethics Committee on Research at the Hospital Saint-Antoine, Paris, by the direct procedure. With respect to the confidentiality of patient records, data handling for the study was authorised by the Commission Nationale d'Informatique et Libertés.

\section{RESULTS}

\section{Population characteristics}

The study initially included 351 consecutive children consulting as outpatients for asthma management and who met the inclusion criteria. Of these, 36 were excluded from analysis as one or more of the cluster analysis parameters were missing. Thus, the final study population included 315 children, 203 $(64.4 \%)$ males. The characteristics of the excluded children did not differ from those of the final sample (data not shown). The characteristics of the final population according to the GINA classification are described in table 1.

\section{Asthma phenotypes according to cluster analysis}

Using the cluster approach, a dendogram was produced and the corresponding scatterplot revealed three clusters of children with shared phenotypic characteristics (fig. 1). These clusters were identified on the basis of: age; BMI; FEV1 level; degree of sensitisation to food or inhaled allergens; systemic eosinophilia, neutrophilia and lymphocytosis (as well as monocytosis although at a borderline level); elevation of the different Ig classes (IgG, IgA, IgM and IgE) in the peripheral blood; asthma control under ICS treatment; and hospitalisation for asthma exacerbation (table 2). The clusters were shown to be stable as the results persisted when variables were excluded, even though the statistical significance of other variables decreased slightly. In addition, the results persisted after altering the sample or subsetting (either for the sample including the 36 patients with missing data for the other variables or by randomly selecting 150 individuals).

\section{Cluster 1}

103 children were included in cluster 1 (table 2). They were more atopic with the highest average number of positive SPTs to both inhaled and food allergens, the highest total IgE level, combined with higher eosinophil values (mean 734, range 650817 cells $\cdot \mathrm{mm}^{-3}$ ) and higher basophil values (mean 42, range 34-50 cells $\cdot \mathrm{mm}^{-3}$ ), and had more maternal or paternal asthma. Duration of asthma was longer than in other clusters ( $>5 \mathrm{yrs}$ in $88 \%$ of children). The FEV1 was slightly lower in this cluster compared with cluster 3. Asthma was more frequently uncontrolled despite high doses of ICS (14\%) in this group,

TABLE 1 Characteristics of the studied population

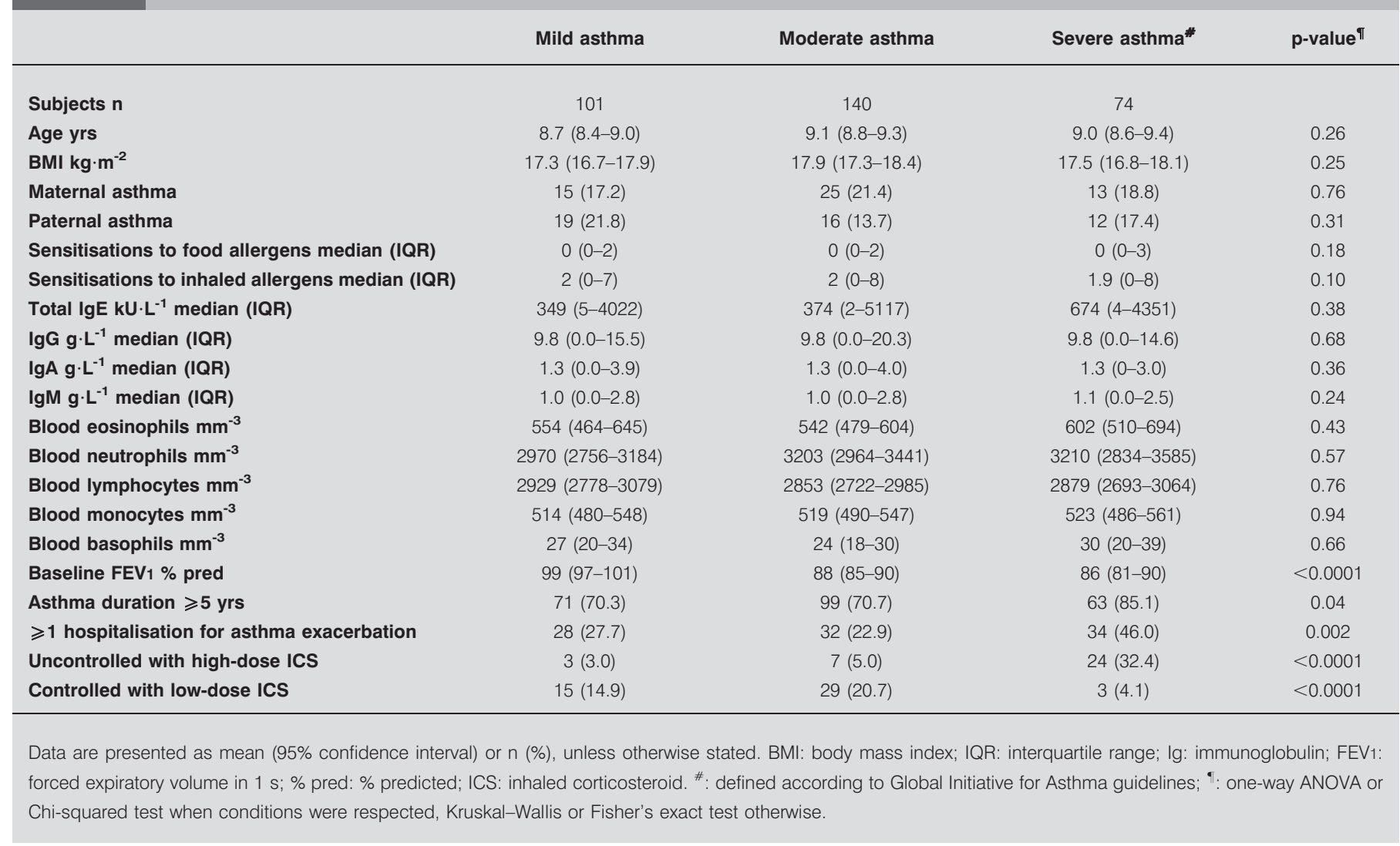




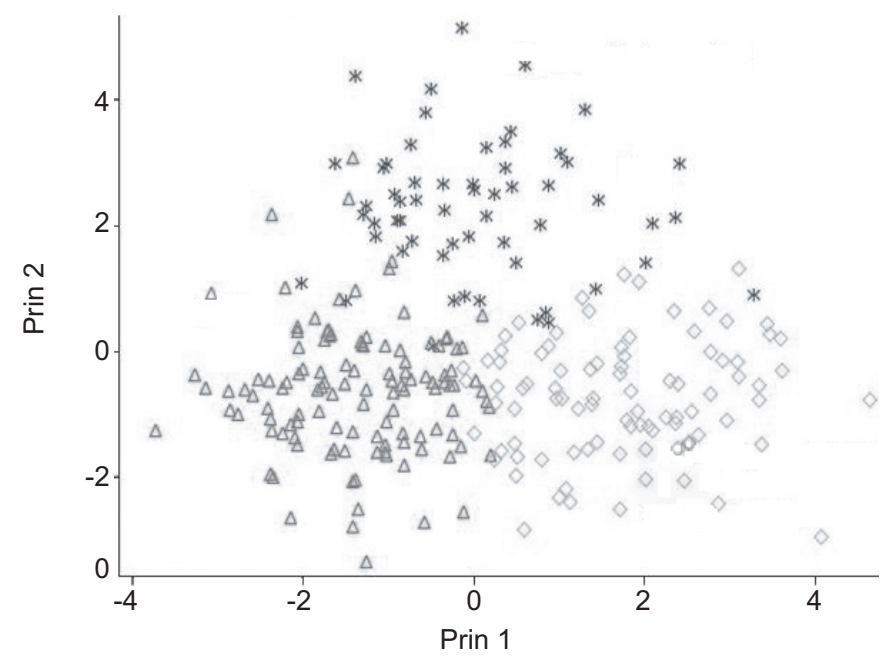

FIGURE 1. Scatterplot for clusters using the criteria of Ward in the entire population of 315 children. Each data point represents a single child. The plot depicts clustering and clear separation of: 103 children with asthma with severe exacerbations and multiple allergies (diamonds); 72 children with severe asthma with bronchial obstruction (stars); and 140 children with mild asthma (triangles). The Principal (Prin) Component Analysis was used for clustering and more severe exacerbation requiring at least one hospitalisation $(65 \%)$ being reported. This asthma phenotype can be termed "asthma with severe exacerbations and multiple allergies".

\section{Cluster 2}

72 children were grouped in this cluster termed "severe asthma with bronchial obstruction" (table 2). The children in this group were significantly older (mean age $10 \mathrm{yrs}$ ) and had the highest BMI (mean 20, range 19-21 $\mathrm{kg} \cdot \mathrm{m}^{-2}$ ). Children in this cluster had a significantly lower baseline pulmonary function in terms of FEV1 (mean 82, range 78-86\% predicted). This phenotype was associated with the most pronounced "neutrophil inflammation" with a non-significant increase in monocyte values (mean 550 , range $511-588$ cells $\cdot \mathrm{mm}^{-3} ; \mathrm{p}=0.08$ ), and a significant increase in absolute neutrophil values (mean 3,423, range $3,082-3,765$ cells $\cdot \mathrm{mm}^{-3}$ ), as well as a significant increase in all classes of $\operatorname{Ig}$ (IgG: mean 11.7, range $11.2-12.3 \mathrm{~g} \cdot \mathrm{L}^{-1}$; IgA: mean 1.8 , range $1.6-1.9 \mathrm{~g} \cdot \mathrm{L}^{-1}$; and IgM: mean 1.3 , range $\left.1.1-1.4 \mathrm{~g} \cdot \mathrm{L}^{-1}\right)$.

\section{Cluster 3}

Cluster 3 was the largest group with 140 children (table 2). This cluster was characterised by the highest average value of FEV1 (mean 97, range 95-100\% pred), less sensitisation to

TABLE 2 Characteristics of children according to cluster analysis in the entire population

\section{Cluster 1: asthma with severe exacerbations and multiple allergies}

Cluster 2: severe asthma with bronchial obstruction

\begin{tabular}{|c|c|c|c|c|}
\hline Subjects $n$ & 103 & 72 & 140 & \\
\hline Age yrs & $8.8(8.5-9.2)$ & $10.3(10.0-10.6)$ & $8.3(8.0-8.5)$ & $<0.0001$ \\
\hline BMI kg $\cdot \mathrm{m}^{-2}$ & $17.1(16.6-17.5)$ & $20.0(19.1-21.0)$ & $16.7(16.3-17.0)$ & $<0.0001$ \\
\hline Maternal asthma & $25(33)$ & $8(13)$ & $16(13)$ & $<0.001$ \\
\hline Paternal asthma & $25(29)$ & $11(18)$ & $11(9)$ & 0.001 \\
\hline Sensitisations to food allergens median (IQR) & $0.3(0.2-0.5)$ & $0.0(0.0-0.0)$ & $0.1(0.0-0.1)$ & $<0.0001$ \\
\hline Sensitisations to inhaled allergens median (IQR) & $3.0(2.6-3.5)$ & $1.9(1.5-2.3)$ & $1.2(1.0-1.5)$ & $<0.0001$ \\
\hline Total IgE $\mathbf{k U} \cdot \mathrm{L}^{-1}$ median (IQR) & $805(657-952)$ & $485(365-605)$ & $450(323-577)$ & $<0.0001$ \\
\hline IgG $\mathbf{g} \cdot \mathrm{L}^{-1}$ median (IQR) & $9.9(9.6-10.4)$ & $11.7(11.2-12.3)$ & $8.6(8.1-19.0)$ & $<0.0001$ \\
\hline $\lg A \mathrm{~g} \cdot \mathrm{L}^{-1}$ median (IQR) & $1.3(1.2-1.4)$ & $1.8(1.6-1.9)$ & $1.1(1.0-1.2)$ & $<0.0001$ \\
\hline $\lg M \mathbf{g} \cdot \mathrm{L}^{-1}$ median (IQR) & $1.1(1.0-1.2)$ & $1.3(1.1-1.4)$ & $0.9(0.9-1.0)$ & 0001 \\
\hline Blood eosinophils $\mathrm{mm}^{-3}$ & $734(650-817)$ & $514(421-607)$ & $454(395-515)$ & $<0.0001$ \\
\hline Blood basophils $\mathrm{mm}^{-3}$ & $42(34-50)$ & $3(4-14)$ & $24(18-23)$ & $<0.0001$ \\
\hline Blood lymphocytes $\mathrm{mm}^{-3}$ & $3036(2889-3182)$ & 3030 (2852-3208) & $2691(2561-2820)$ & $<0.001$ \\
\hline Blood neutrophils $\mathrm{mm}^{-3}$ & 2767 (2540-2993) & $3423(3082-3765)$ & $3250(3009-3492)$ & 0.001 \\
\hline Blood monocytes $\mathrm{mm}^{-3}$ & $505(474-536)$ & $550(511-588)$ & $515(483-541)$ & 0.08 \\
\hline Baseline FEV $1 \%$ pred & 89 (86-92) & $82(78-86)$ & $97(95-100)$ & $<0.0001$ \\
\hline Asthma duration $\geqslant 5$ yrs & $91(88)$ & $49(68)$ & $93(66)$ & $<0.001$ \\
\hline$\geqslant 1$ hospitalisation for asthma exacerbation & $67(65)$ & $7(10)$ & $20(14)$ & $<0.0001$ \\
\hline Uncontrolled with high-dose ICS & $15(15)$ & $6(8)$ & $13(9)$ & $<0.0001$ \\
\hline Controlled with low-dose ICS & $9(9)$ & $2(3)$ & $36(26)$ & $<0.0001$ \\
\hline
\end{tabular}

Data are presented as mean (95\% confidence interval) or $\mathrm{n}(\%)$, unless otherwise stated. BMI: body mass index; IQR: interquartile range; Ig: immunoglobulin; FEV1: forced expiratory volume in $1 \mathrm{~s}$; \% pred: \% predicted; ICS: inhaled corticosteroid. ${ }^{\#}$ : one-way ANOVA or Chi-squared test when conditions were respected, Kruskal-Wallis or Fisher's exact test otherwise. 
inhaled or food allergens, and no elevation of either blood cells or Ig. In this population, asthma was more often controlled with low doses of ICS than in the other clusters (26\% versus $9 \%$ and $3 \%$ in clusters 1 and 2, respectively). This phenotype can be summarised as "mild asthma".

\section{Asthma phenotypes and asthma severity}

There was no relationship between severity related to GINA classification and the clusters with a heterogeneous distribution of severity classically defined in each cluster (fig. 2).

\section{DISCUSSION}

Our study identified two novel severe phenotypes in childhood asthma compared to a mild asthma phenotype. These two novel severe asthma phenotypes are: 1) asthma with severe exacerbations, and 2) multiple allergies and severe asthma with bronchial obstruction.

Similar to a previous report [9], in our study all the clusters of childhood asthma presented with atopic features, but the magnitude and the nature of allergic sensitisation differed among clusters. Moreover, the nature of systemic inflammation, eosinophil-basophil type, neutrophil type or no inflammation was different amongst the groups and this difference in asthmatic children has not been previously described.

\section{Asthma with severe exacerbations and multiple allergies}

This type of severe asthma is associated with inflammation that is predominantly "allergic" (with eosinophil and basophil cells) in combination with multiple allergic sensitisations and elevated total IgE. An earlier study by our group in asthmatic children showed a link between intra-alveolar eosinophilia and atopy [15]. Moreover, it is known that blood eosinophilia is closely correlated to eosinophil inflammation in the deep lung tissues [16]. This phenotype is associated with severe exacerbations requiring hospitalisations and uncontrolled asthma despite high doses of ICS. Many studies have confirmed that asthma at risk of

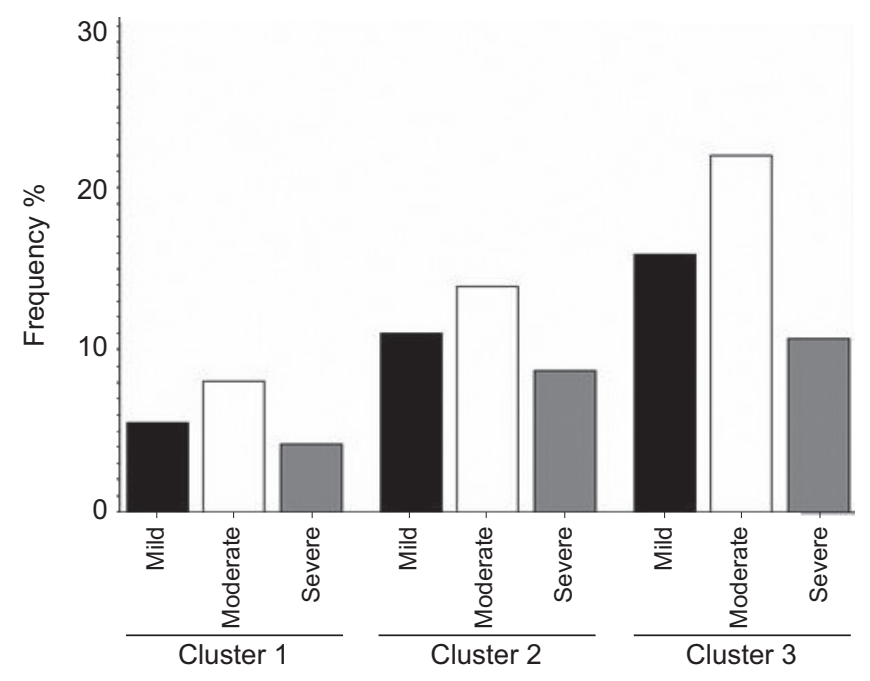

FIGURE 2. Frequency of children with mild, moderate and severe asthma defined by Global Initiative for Asthma (GINA) guidelines. The sum of all clusters (divided by GINA classification in each cluster) is equal to $100 \%$. Each GINA class was heterogeneously distributed in each cluster without predominance of severity in any cluster. severe exacerbations or difficult to control asthma is mainly associated with allergic asthma in children $[17,18]$. Severe acute asthma requiring intensive care was more frequently found in cases of food allergy [19, 20]. ROBERTS et al. [19] compared 19 asthmatic children ventilated for severe exacerbation with 38 mild asthmatic children and showed that the two independent risk factors were the severity of asthma (OR 5.89, 95\% CI 1.0632.61) and food allergy (OR 9.85, 95\% CI 1.04-93.27).

Finally, in the phenotype with a higher duration of asthma (frequency $>5$ yrs), FEV1 values were slightly lower compared with the FEV1 values observed in the mild asthma cluster. This was also reported in the Severe Asthma Research Program (SARP) study [9], in which the most allergic children had severe exacerbations despite a high level of therapy but did not have severely decreased FEV1 values. Indeed, severe exacerbations could be responsible for the more rapid decline in lung function [21].

\section{Severe asthma with bronchial obstruction}

This phenotype of severe asthma is characterised in our study by a significant decrease in FEV1 (mean 82, range 78-86\% pred) even if it remains within the accepted limits of normal, as in other studies in severe asthmatic children $[22,23]$. This finding is probably due to the fact that the normal value of FEV1 in children at school age is $>90 \%$, as in our cluster 3 in which the mean value of FEV1 was $97 \%$ pred (range $95-100 \%$ pred).

The cluster was also characterised by a significant elevation in neutrophils in peripheral blood in comparison with the other clusters. Many studies have shown an association between the severity of asthma and neutrophilic inflammation detected by induced sputum [24, 25]. Furthermore, our study supports the findings of SIROUx et al. [26] who reported that the phenotype of "active treated allergic childhood-onset asthma" is associated with blood eosinophilia, while "active treated adult-onset asthma" is associated with blood neutrophils. Systemic neutrophilic inflammation is mainly described in inflammation of infectious type. In the same way, elevation of Ig has been related to an inflammation in asthma originating from a bacterial colonisation. It is well known that this inflammation type induces uncontrolled asthma [27] and corticosteroid resistance [28] and, therefore, probably increases the risk of tissue remodelling, which would explain the lower FEV1 values in this phenotype.

This phenotype was also associated with a higher BMI. The association between obesity and severe asthma has been reported in several studies [29-31]. HALDAR et al. [30] showed that a distinct cluster of asthmatic subjects with high BMI was associated with symptomatic asthma without eosinophilic inflammation. The study by MOORE et al. [31] also found a cluster of asthma associated with a moderate reduction in FEV1 in females who were overweight with delayed onset. Finally, our results are consistent with the SARP study, including the fact that asthma severity is not related to a markedly low FEV1 or to the GINA severity score [9].

The main limitation to our study lies in the fact that all the patients were recruited from one centre, and this of course could represent a geographical bias. Nevertheless, while twothirds of the asthmatic children were from Paris and the surrounding area ( $>10$ million inhabitants), the remaining onethird live in regions throughout France, which limits this potential bias. However, our results cannot be generalised in 
another population, especially with greater ethnic diversity. Moreover, our sample is the largest among those used in previous similar studies and our results fit the clinical context. An important strength of our study is that the unsupervised cluster analysis identified the clusters without any a priori assignment and the stability of the clusters was tested. This has rarely been carried out before for children. Furthermore, our investigation is unlike previous cluster analyses of asthma as we included blood inflammatory markers and demonstrate that this constitutes an important feature of asthma.

In conclusion, this large study of asthmatic children differentiates two novel severe asthma phenotypes. We would suggest that these phenotypes be included in recommendations on the management of severe asthma in children in order to optimise treatment strategies, i.e. anti-allergic drugs such as anti-IgE or anti-interleukin-5 for children with the severe allergic phenotype, and anti-neutrophil drugs such as macrolides for those with the obstructive phenotype; thus, potentially improving long-term outcome. Further studies investigating inflammation in deep lung tissues are required to confirm these results.

\section{SUPPORT STATEMENT}

This study was supported by a research grant from the Epidemiological Research Group, Laboratoire Novartis (Paris, France).

\section{STATEMENT OF INTEREST}

A statement of interest for J. Just and the study itself can be found at www.erj.ersjournals.com/site/misc/statements.xhtml

\section{ACKNOWLEDGEMENTS}

The authors would like to thank V. Le Gros (Clinical Research and Development, Novartis, Paris, France) for obtaining the funding for the study.

\section{REFERENCES}

1 Ronchetti R, Villa MP, Barreto M, et al. Is the increase in childhood asthma coming to an end? Findings from three surveys of schoolchildren in Rome, Italy. Eur Respir J 2001; 17: 881-886.

2 Yeh KW, Fang W, Huang JL. Increasing the hospitalization of asthma in children not in adults - from a national survey in Taiwan 1996-2002. Pediatr Allergy Immunol 2008; 19: 13-19.

3 Wenzel SE. Asthma: defining of the persistent adult phenotypes. Lancet 2006; 368: 804-813.

4 Moore WC, Peters SP. Update in asthma 2006. Am J Respir Crit Care Med 2007; 175: 649-654.

5 Martinez FD, Wright AL, Taussig LM, et al. Asthma and wheezing in the first six years of life. The Group Health Medical Associates. N Engl J Med 1995; 332: 133-138.

6 Miranda C, Busacker A, Balzar S, et al. Distinguishing severe asthma phenotypes: role of age at onset and eosinophilic inflammation. J Allergy Clin Immunol 2004; 113: 101-108.

7 Zeiger RS, Dawson C, Weiss S. Relationships between duration of asthma and asthma severity among children in the Childhood Asthma Management Program (CAMP). J Allergy Clin Immunol 1999; 103: 376-387.

8 Brand PL, Baraldi E, Bisgaard H, et al. Definition, assessment and treatment of wheezing disorders in preschool children: an evidence-based approach. Eur Respir J 2008; 32: 1096-1110.

9 Fitzpatrick AM, Teague WG, Meyers DA, et al. Heterogeneity of severe asthma in childhood: confirmation by cluster analysis of children in the National Institutes of Health/National Heart, Lung, and Blood Institute Severe Asthma Research Program. J Allergy Clin Immunol 2011; 127: 382-389.
10 Urbano FL. Review of the NAEPP 2007 Expert Panel Report (EPR-3) on Asthma Diagnosis and Treatment Guidelines. J Manag Care Pharm 2008; 14: 41-49.

11 Beydon N, Davis SD, Lombardi E, et al. An official American Thoracic Society/European Respiratory Society statement: pulmonary function testing in preschool children. Am J Respir Crit Care Med 2007; 175: 1304-1345.

12 Bateman ED, Hurd SS, Barnes PJ, et al. Global strategy for asthma management and prevention: GINA executive summary. Eur Respir J 2008; 31: 143-178.

13 Hennig C. Cluster-wise assessment of cluster stability. Comp Stat Data Anal 2007; 52: 258-271.

14 Elliott AC, Hynan LS. A SAS(®) macro implementation of a multiple comparison post hoc test for a Kruskal-Wallis analysis. Comput Methods Programs Biomed 2011; 102: 75-80.

15 Just J, Nicoloyanis N, Chauvin M, et al. Lack of eosinophilia can predict remission in wheezy infants? Clin Exp Allergy 2008; 38: 767-773.

16 Bousquet J, Chanez P, Lacoste JY, et al. Eosinophilic inflammation in asthma. N Engl J Med 1990; 323: 1033-1039.

17 Carroll WD, Lenney W, Child F, et al. Asthma severity and atopy: how clear is the relationship? Arch Dis Child 2006; 91: 405-409.

18 O'Driscoll BR, Hopkinson LC Denning DW. Mold sensitization is common amongst patients with severe asthma requiring multiple hospital admissions. BMC Pulm Med 2005; 5: 4.

19 Roberts G, Patel N, Levi-Schaffer F, et al. Food allergy as a risk factor for life-threatening asthma in childhood: a case-controlled study. J Allergy Clin Immunol 2003; 112: 168-174.

20 Vogel NM, Katz HT, Lopez R, et al. Food allergy is associated with potentially fatal childhood asthma. J Asthma 2008; 45: 862-866.

21 Donaldson GC, Seemungal TA, Bhowmik A, et al. Relationship between exacerbation frequency and lung function decline in chronic obstructive pulmonary disease. Thorax 2002; 57: 847-852.

22 Harver A, Schwartzstein RM, Kotses H, et al. Descriptors of breathlessness in children with persistent asthma. Chest 2011; 139: 832-838.

23 Mahut B, Delclaux C, Tillie-Leblond I, et al. Both inflammation and remodeling influence nitric oxide output in children with refractory asthma. J Allergy Clin Immunol 2004; 113: 252-256.

24 The ENFUMOSA cross-sectionnal European multicentre study of the clinical phenotype of chronic severe asthma. European Network for Understanding Mechanisms of Severe Asthma. Eur Respir J 2003; 22: 470-477.

25 Wang F, He XY, Baines KJ, et al. Different inflammatory phenotypes in adults and children with acute asthma. Eur Respir J 2011; 38: 567-574.

26 Siroux V, Basagana X, Boudier A, et al. Identifying adult asthma phenotypes using a clustering approach. Eur Respir J 2011; 38: 310-317.

27 Kowalski ML, Cieslak M, Perez-Novo CA, et al. Clinical and immunological determinants of severe/refractory asthma (SRA): association with Staphylococcal superantigen-specific IgE antibodies. Allergy 2011; 66: 32-38.

28 Huang YJ, Nelson CE, Brodie EL, et al. Airway microbiota and bronchial hyperresponsiveness in patients with suboptimally controlled asthma. J Allergy Clin Immunol 2011; 127: 372-381.

29 Peters JI, McKinney JM, Smith B, et al. Impact of obesity in asthma: evidence from a large prospective disease management study. Ann Allergy Asthma Immunol 2011; 106: 30-35.

30 Haldar P, Pavord ID, Shaw DE, et al. Cluster analysis and clinical asthma phenotypes. Am J Respir Crit Care Med 2008; 178: 218-224.

31 Moore WC, Meyers DA, Wenzel SE, et al. Identification of asthma phenotypes using cluster analysis in the Severe Asthma Research Program. Am J Respir Crit Care Med 2010; 181: 315-323. 